\title{
A PESSOA SUBMETIDA A ARTROPLASTIA TOTAL DA ANCA POR COXARTROSE
}

THE PERSON UNDERGOING TOTAL HIP ARTHROPLASTY DUE TO COXARTHROSIS: CASE REPORT

LA PERSONA Que SE SOMETE A UMA PRÓTESIS TOTAL DE CADERA POR COXARTROSIS: RELATO DE CASO

DOI 10.33194/rper.2021.167; Data de Receção: 2021/04/26; Data de Aceitação: 2021/06/20

Pedro Dias ${ }^{1}\left(\mathbb{D}\right.$; Rogério Ferrinho Ferreira ${ }^{2} \mathbb{D}$; Patrícia Messias ${ }^{1}(\mathbb{D}$

${ }^{1}$ Centro Hospitalar Universitário do Algarve, Faro, Portugal; ${ }^{2}$ Escola Superior de Saúde de Beja, Beja, Portugal

\author{
Autor correspondente: Pedro Dias, pedrodias_vrsa@hotmail.com
}

\section{RESUMO}

Introdução: As modificações biológicas decorrentes do envelhecimento podem acarretar problemas nas articulações, sendo a coxartrose uma das mais comuns. Decorrente das consequências e do modo como afeta a qualidade de vida das pessoas, a artroplastia total da anca surge como uma solução. Neste sentido, destaca-se a intervenção do Enfermeiro Especialista em Enfermagem de Reabilitação como o profissional capaz de potenciar a funcionalidade e maximizar a independência da pessoa.

Objetivo: Identificar os benefícios da intervenção do Enfermeiro Especialista em Enfermagem de Reabilitação, no período pré e pós-operatório de uma pessoa submetida a artroplastia total da anca por coxartrose.

Metodologia: Estudo de caso descritivo único, fundamentado nas guidelines CARE (CAse REport). Implementou-se um programa de reabilitação específico e direcionado para a pessoa em estudo, baseado nos diagnósticos identificados, com particular interesse na Reeducação Funcional Motora.

Resultados: Verificou-se um aumento da força muscular e da amplitude articular no membro intervencionado, melhorias relativas ao equilíbrio corporal e independência na realização das atividades de vida diária e uma diminuição do risco de queda e intensidade da dor.

Conclusão: A capacidade funcional melhorou após intervenção cirúrgica e implementação do programa de enfermagem de reabilitação.

DESCRITORES: Enfermagem de Reabilitação; Reeducação Funcional Motora; Artroplastia Total da Anca; Coxartrose

\section{ABSTRACT}

Introduction: Biological changes duo to aging can cause joint problems and coxarthrosis is one of the most common. Due to the consequences and the way it affects people's quality of life, total hip arthroplasty appears as one of the solutions. Thus, the rehabilitation nurse stands out as the professional capable of enhancing functionality and maximizing the person's independence.

Objective: Identify the benefits of the rehabilitation nurse's intervention, in the pre and postoperative period of a person undergoing total hip arthroplasty due to coxarthrosis.

Methodology: Unique descriptive case study, based on the CARE guidelines (CAse REport). A specific rehabilitation program was implemented and targeted at the person under study, based on the diagnoses identified and giving particular interest to Functional Motor Reeducation.

Results: There was an increase in muscle strength and range of motion in the surgically operated limb, improvements in body balance and independence in performing activities of daily living and a decrease in the risk of falling and pain intensity.

Conclusion: Functional capacity improved after surgical intervention and a rehabilitation nursing program.

DESCRIPTORS: Rehabilitation Nursing; Functional Motor Reeducation; Total Hip Arthroplasty; Coxarthrosis. 
Introducción: Los cambios biológicos en el envejecimiento causan problemas articulares, como la coxartrosis. Por las consecuencias y cómo afecta la calidad de vida, la prótesis total de cadera surge como una solución. Así, se destaca la intervención del Enfermero Especialista en Enfermería de Rehabilitación como el profesional que promueve la funcionalidad y maximiza la independencia.

Objetivo: Identificar los beneficios de la intervención del Enfermero Especialista en Enfermería de Rehabilitación, en el pre y postoperatorio de una persona que se somete a una prótesis total de cadera por coxartrosis.

Metodología: Relato de caso descriptivo único, apoyado en las directrices CARE (CAse REport). Se implementó un programa de rehabilitación específico y dirigido a la persona en estudio, teniendo en cuenta los diagnósticos identificados, con especial interés en la Reeducación Funcional Motora.

Resultados: Hubo un aumento de la fuerza muscular y amplitud de movimiento en el miembro operado, mejoras en el equilibrio corporal y en la independencia para realizar las actividades de la vida diaria, y una disminución del riesgo de caídas y del dolor.

Conclusión: La capacidad funcional mejoró después de una intervención quirúrgica y un programa de rehabilitación.

DESCRIPTORES: Enfermería de Rehabilitación; Reeducación Funcional Motora; Prótesis Total de Cadera; Coxartrosis

\section{INTRODUÇÃO}

O envelhecimento, processo de mudança natural e progressivo, transversal a todas as sociedades, evidencia no idoso as doenças, co morbilidades, incapacidades e dependências ${ }^{(1)}$. Em 2020, apenas $15,6 \%$ das pessoas idosas portuguesas percecionavam de forma positiva a sua saúde, colocando Portugal na lista dos países da União Europeia com a perceção de saúde mais depreciativa( ${ }^{(2)}$. É de destacar que ainda em $2020,73,8 \%$ da população com 65 ou mais anos tinha associado a existência de doenças crónicas à sua perceção de saúde, sendo as mulheres e a população idosa $(60,8 \%)$ os que referem ter, com mais frequência, alguma limitação na execução de atividades habituais ${ }^{(2)}$. Estes dados demonstram a necessidade dos cuidados de enfermagem de reabilitação irem ao encontro das necessidades reais das pessoas, nomeadamente na capacitação para a independência na realização das atividades de vida diária.

O fenómeno do envelhecimento provoca modificações biológicas, tais como a osteoporose, que se evidencia principalmente nas mulheres como um problema que afeta a independência funcional ${ }^{(3)}$. Cerca de 3-6\% da população europeia sofre de sintomas relacionados com a degeneração osteoarticular, dados estes que irão aumentar no futuro devido ao envelhecimento populacional ${ }^{(4)}$. Quando a dor e o sofrimento sentido pela pessoa expressam limitações que a impedem de desempenhar as suas atividades de vida diária, pode ser proposta a artroplastia total da anca, na qual se implanta uma prótese na diáfise femoral, constituída por cabeça, colo e extremidade (componente femoral), e outra no osso ilíaco (componente acetabular) ${ }^{(3)}$. Assim, a artroplastia destaca-se com o tratamento de eleição nos estadios finais da osteoartrite da anca, sendo que existem vários estudos que mostram o impacto negativo na qualidade de vida destas pessoas. Contudo, ainda que seja dado valor à reabilitação pós-operatória, pouco se sabe acerca da eficácia dos exercícios atendendo ao tipo, frequência, intensidade e duração do programa de reabilitação ${ }^{(4)}$.

Ressalva-se desta forma a importância dos cuidados de enfermagem desde o período pré-operatório até ao pósoperatório, sendo que o tratamento da pessoa sujeita a esta cirurgia não termina com a protetização da anca, devendo continuar para o resto da vida. Os cuidados prestados devem ser assim, adaptados e de acordo com uma visão holística da pessoa ${ }^{(3)}$.

O sucesso da intervenção cirúrgica de artroplastia total da anca vai depender da assimilação de conhecimentos e dos ensinos fornecidos à pessoa, bem como da capacidade do enfermeiro em integrar esses cuidados nas atividades de vida diária, de modo a promover a preservação da articulação(3).

Num estudo envolvendo 54 pessoas submetidas a artroplastia total da anca, destacaram-se os principais défices resultantes desta cirurgia, tais como a diminuição da força muscular, do equilíbrio postural e da marcha, tendo-se verificado que, embora a marcha numa condição de descanso não influenciasse a capacidade muscular do doente, o aumento da força muscular decorrente de uma realização exigente das atividades de vida diária, limitou o equilíbrio corporal $^{(5)}$. Outro estudo refere que uma mobilização articular direcionada, juntamente com treino de força muscular, além de bem tolerados pela pessoa, mostram melhorias na amplitude de movimento da articulação coxofemoral e no desempenho da marcha ${ }^{(6)}$. É nesse sentido que a atuação do Enfermeiro Especialista em Enfermagem de Reabilitação (EEER) deve ser centrada, nomeadamente no desenvolvimento deste tipo de programas de treino motor e de atividades de vida diária, baseados em e para criar evidência científica.

O EEER, dotado de competências específicas necessárias para capacitar a pessoa com limitação da atividade, bem como para maximizar a sua funcionalidade, atendendo às capacidades remanescentes, contribui para a minimização das sequelas resultantes de uma nova condição e permite que a pessoa readquira o seu máximo de funcionalidade possível. Este profissional elabora planos de enfermagem de reabilitação diferenciados, atendendo aos problemas reais e potencias identificados na avaliação inicial. Nesse plano de enfermagem constam, não só o diagnóstico precoce 
realizado pelo EEER, como as ações e intervenções terapêuticas que irão ser realizadas na etapa da execução, e que irão permitir recuperar ou manter a funcionalidade da pessoa cuidada. Este planeamento só é possível pelo facto deste profissional possuir experiência acrescida e um alto nível de conhecimentos, que the permite uma tomada de decisão fundamentada. Numa fase final, é realizada uma avaliação dos resultados, tendo em conta a correta identificação dos problemas e objetivos, planeamento das intervenções e sua execução( ${ }^{(7)}$.

Desta forma, o EEER possui competências que o permitem implementar programas de reabilitação dirigidos à pessoa com alterações do foro músculo-esquelético, com o intuito de promover a sua independência funcional, bem como a participação social. Os programas de reabilitação implementados pelo EEER assumem assim uma elevada importância, nomeadamente no idoso, uma vez que é possível prevenir complicações, como o risco de queda ou os problemas cardiovasculares, a partir de uma reabilitação precoce com promoção da força muscular e funcionalidade, que possibilitam preservar a sua independência o maior tempo possível ${ }^{(6)}$.

Neste contexto, o objetivo de âmbito geral deste estudo centra-se em identificar os benefícios de um programa de reabilitação, no período pré e pós-operatório de uma pessoa idosa submetida a artroplastia total da anca, dando especial atenção à reeducação funcional motora e ao treino de atividades de vida diária. Os objetivos específicos caracterizam-se por: reconhecer as modificações funcionais e músculo-esqueléticas na pessoa, mediante utilização de instrumentos de avaliação; definir os diagnósticos específicos de enfermagem reabilitação; implementar um programa de treino motor e de atividades de vida diária específico para a pessoa em estudo; avaliar os resultados obtidos com a implementação do plano de cuidados individualizado de enfermagem de reabilitação à pessoa submetida a artroplastia total da anca.

\section{METODOLOGIA}

Um estudo de caso descritivo único, encontra-se integrado numa metodologia de investigação, usada com frequência na compreensão de determinados fenómenos ${ }^{(8)}$ e que permite relatar a história clínica da pessoa para propósitos científicos e educacionais, ou seja, possibilita reconhecer doenças novas ou raras, avaliar os efeitos vantajosos ou perigosos de determinada intervenção e promover a educação e evolução da medicina ${ }^{(9)}$. Ao ser útil em investigação, surge como uma forma de produzir evidência científica ${ }^{(8 ; 9)}$.

O presente estudo de caso baseia-se nas diretrizes CAse REport (CARE), que fornecem claras indicações em relação aos vários itens que constituem um estudo de caso. De forma organizada e lógica foram cumpridos os diversos critérios da checklist, tendo sido realizadas adaptações quando necessário, de modo a adequar ao caso em estudo(9).

Torna-se importante destacar que foram respeitados os princípios legais e que foi adotada uma conduta ética para realizar esta investigação de enfermagem, recorrendo para tal às recomendações da Declaração de Helsínquia, da Convenção de Oviedo, do International Committee of Mecical Journal Editors e do Committee on Publication Ethics. Ainda se torna relevante ressalvar que o consentimento informado, livre e esclarecido foi obtido antes de se dar início à colheita de dados e que o presente estudo de caso foi elaborado e desenvolvido no contexto de um estágio académico, autorizado pelas instituições intervenientes, nomeadamente uma Instituição de Ensino Superior e um Centro Hospitalar. O projeto foi aceite pela comissão de ética da Instituição de Ensino Superior.

Os diagnósticos foram baseados na linguagem CIPE versão $2015^{(10)}$ e no Padrão Documental dos Cuidados de Enfermagem da Especialidade de Enfermagem de Reabilitação( ${ }^{(11)}$. 0 programa de reabilitação foi implementado desde o dia 19 até ao dia 26 de novembro. Os dados colhidos foram analisados quantitativamente, tendo sido selecionados instrumentos de avaliação para assegurar a medição de determinadas funções da pessoa.

Desta forma, implementou-se um programa de reabilitação específico e direcionado para a pessoa em estudo, baseado nos diagnósticos identificados, com particular interesse na Reeducação Funcional Motora.

\section{Avaliação Inicial e História Clínica}

Utente caucasiana do sexo feminino de 67 anos, com $71 \mathrm{~kg}$ e $1,63 \mathrm{~m}$ de altura. Reformada ex-cabeleireira, com antecedentes pessoais de asma e ausência de alergias medicamentosas e/ou alimentares. Reside numa casa sem escadas e mora com uma das duas filhas, elemento que irá prestar cuidados / apoio aquando da alta. No domicílio, embora independente nas atividades de vida diária, realizava-as com bastante dificuldade devido à dor sentida, o que influenciava de forma negativa a sua qualidade de vida.

A medicação que realizava no domicílio em SOS era Maizar Diskus (50 microgramas de salmeterol + 100 microgramas de propionato de fluticasona) e medicação analgésica que não soube especificar.

A admissão no serviço de Ortopedia do Centro Hospitalar ocorreu a 19/11/2020, com o diagnóstico de coxartrose esquerda, para cirurgia eletiva, que só se viria a realizar a 21/11/2020. Em relação aos exames pré-operatórios, foram realizadas análises sanguíneas, que se encontravam dentro dos valores de referência; eletrocardiograma, com ritmo sinusal; e radiografia do tórax e bacia (Figura 1). A senhora foi também submetida a uma avaliação por parte do anestesista, obtendo um risco cirúrgico de ASA II (American Society of Anestesiology), indicativo de doença 
sistémica leve. No dia $21 / 11 / 2020$, foi submetida a artroplastia total da anca esquerda, sob raquianestesia, tendo o pós-operatório decorrido sem intercorrências e com controlo radiográfico da bacia após a cirurgia (Figura 2).

No internamento apresentava a seguinte medicação prescrita: Enoxaparina sódica 40mg SC 17h; Paracetamol 1g PO 7/15/23h; Metamizol magnésico 2g EV 8/8h SOS; Captopril 25mg SL 8/8h SOS; Pantoprazol 20mg PO 7h; Salbutamol $100 \mathrm{yg} /$ dose, Suspensão pressurizada para inalação, $8 / 8 \mathrm{~h}$ SOS.

No dia 26/11/2020, a senhora tem critérios para alta clínica e para dar continuidade aos cuidados iniciados no internamento, no seu domicílio, com apoio da filha com quem reside. Desta forma, para dar suporte à continuidade de cuidados, foi elaborada a nota de alta do EEER.

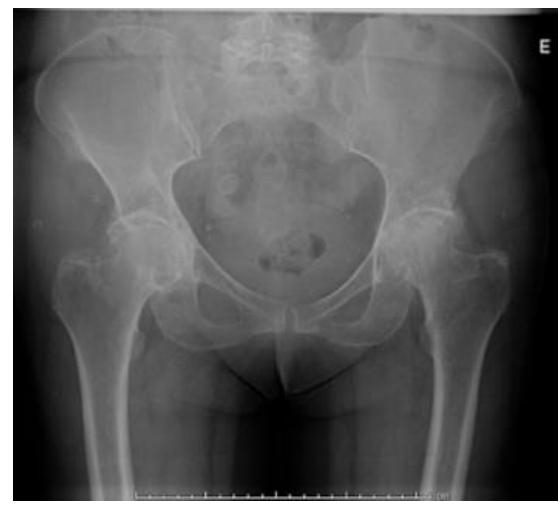

Figura 1: Raio-X da bacia pré-operatório.

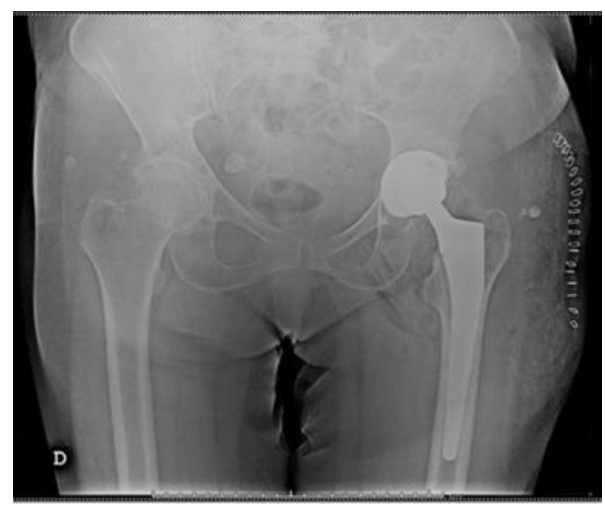

Figura 2: Raio-X da bacia pós-operatório.

\section{Exame físico - 19/11/2020}

Utente consciente, orientada no tempo, espaço e pessoa, e colaborante. Com mucosas coradas e hidratadas, anictérica e acianótica, bem como eupneica, sem síndrome de dificuldade respiratória e com $\mathrm{SpO}_{2} 96 \%$ a ar ambiente. À auscultação pulmonar, evidenciou-se murmúrio vesicular mantido bilateralmente, sem ruídos adventícios. 0 abdómen apresentava-se com timpanismo normal, mole, depressível e indolor à palpação. Sem lesões cutâneas e sem sinais inflamatórios na área a ser intervencionada.

\section{Diagnósticos de Enfermagem de Reabilitação}

O processo de reabilitação em enfermagem enquadra a tomada de decisão em diversas etapas, sendo elas: a colheita de dados ou avaliação inicial, a identificação das problemáticas evidenciadas, a definição de objetivos alcançáveis, a escolha das intervenções tendo em conta a pessoa como um ser holístico, a avaliação dos resultados e a preparação para a alta. Todo este processo é passível de ser reavaliado até que se possa dar por finalizado o processo de reabilitação(12).

Neste sentido, e atendendo às principais preocupações da senhora e às necessidades identificadas, foram estabelecidos os diagnósticos e intervenções de Enfermagem de Reabilitação que se seguem, tendo por base a linguagem CIPE versão $2015^{(10)}$ e o Padrão Documental dos Cuidados de Enfermagem da Especialidade de Enfermagem de Reabilitação(11):

Diagnóstico 1: Conhecimento sobre a doença ausente, relacionada com a intervenção cirúrgica;

Diagnóstico 2: Dor músculo-esquelética presente à mobilização do membro inferior esquerdo;

Diagnóstico 3: Movimento muscular diminuído no membro inferior esquerdo;

Diagnóstico 4: Equilíbrio corporal comprometido;

Diagnóstico 5: Capacidade para transferir-se comprometida em grau moderado;

Diagnóstico 6: Andar com auxiliar de marcha comprometido;

Diagnóstico 7: Alto risco de queda presente.

De modo a implementar as intervenções relacionadas com os diagnósticos elaborados, utilizaram-se os seguintes materiais e recursos disponíveis: goniómetro, bastão, andarilho, cadeira de rodas/cadeirão, barras laterais na casa de banho, roupa pessoal confortável e sapatos fechados antiderrapantes.

A escolha dos instrumentos de avaliação de uma certa função deve assentar no pressuposto que é uma medida válida para a função estudada e possuir uma fiabilidade adequada, sendo que os resultados obtidos devem ser sensíveis o suficiente para revelarem alterações clínicas significativas ${ }^{(13)}$. Desta forma, os instrumentos selecionados foram: a escala de coma Glasgow, a escala de força de Medical Research Council, a escala simples de equilíbrio, a escala de Barthel, a escala de Morse e a escala numérica da dor. 
A escala de coma de Glasgow avalia o estado de consciência da pessoa, revelando-se um elemento importante para o estudo de caso em questão, visto que se planeiam intervenções direcionadas para a assimilação de conhecimento, adequadas para uma pessoa consciente e orientada, que tenha capacidades para continuar no domicílio os cuidados instruídos no internamento ${ }^{(14)}$.

De seguida, torna-se importante na pessoa com alterações do foro músculo-esquelético avaliar o grau de força dos diversos segmentos corporais para que se consiga implementar intervenções, tais como o levante, o treino de marcha ou o treino de atividades de vida diária. Utilizou-se a escala de força de Medical Research Council com o objetivo de se avaliar a força de todos os segmentos corporais da pessoa, de modo a prosseguir-se em segurança para a realização de intervenções específicas do Enfermeiro Especialista em Enfermagem de Reabilitação ${ }^{(15)}$.

Para que se possa realizar um levante correto e em segurança, é necessário primeiro averiguar se a pessoa possui equilíbrio na posição de sentada (estático e dinâmico), e em caso afirmativo proceder-se ao levante. Na posição de pé consegue-se verificar se a pessoa detém equilíbrio ortostático (estático e dinâmico), sendo que, caso algum destes se encontre diminuído ou ausente, fornece dados para que o Enfermeiro Especialista em Enfermagem de Reabilitação possa atuar e intervir junto da pessoa. A escala simples do equilíbrio constitui assim um instrumento imprescindível na reeducação funcional motora.

Com o intuito de realizar treino de atividades de vida diária para que a pessoa possa otimizar e progredir na situação clínica, recorreu-se à escala de Barthel, que avalia o grau de independência da pessoa na execução de dez atividades básicas de vida diária ${ }^{(16)}$.

A escala de quedas de Morse também se constituiu como um instrumento de relevância a ser utilizado, uma vez que a prevenção de quedas e dos danos resultantes é, a nível mundial, um dos objetivos de saúde pública, sendo que as quedas constituem, não só uma principal causa de internamento, como de morte ${ }^{(17 ; 18)}$. Neste sentido, e associado ao equilíbrio comprometido que a pessoa com alterações do foro músculo-esquelético pode apresentar, destaca-se a importância da escolha e aplicação desta escala.

Por fim, para que se consiga iniciar e dar continuidade a um programa de reeducação funcional motora, torna-se essencial a constante avaliação da dor e tolerância da pessoa às intervenções implementadas, e daí o recurso à escala numérica da dor ${ }^{(19)}$.

\section{Plano de Intervenção - 19/11/2020 a 26/11/2020}

Diagnóstico 1: Conhecimento sobre a doença ausente, relacionada com a intervenção cirúrgica.

Objetivos: Fomentar na pessoa a aquisição de conhecimentos face ao processo de recuperação; obter a colaboração da pessoa no período pós-operatório; reduzir complicações pós-operatórias; otimizar estado funcional da pessoa; diminuir tempo de internamento.

Intervenções: Solicitar a colaboração da pessoa; Explicar à pessoa os benefícios da reabilitação precoce no período pós-operatório; Alertar para sinais e sintomas de possíveis complicações (calor, edema, ardor do membro inferior, febre); Sensibilizar e instruir a importância da frequente alternância de decúbitos no leito, respeitando os limites e o risco de luxação da articulação no pós-operatório; Ensinar movimentos potencialmente luxantes; Incentivar à mobilização articular dentro das amplitudes toleradas, para melhor adesão da pessoa ao programa de reabilitação no período pós-operatório; Instruir o levante do leito; Ensinar sobre medidas de segurança (manutenção de auxiliares de marcha e apoio na marcha e uso de sapato antiderrapante fechado durante a marcha); Instruir marcha a 3 pontos com recurso a auxiliar de marcha (andarilho): andarilho, membro inferior esquerdo operado, membro inferior direito; Instruir acerca dos movimentos musculares e articulares; Instruir e treinar técnicas de reeducação funcional respiratória (consciencialização e dissociação dos tempos respiratórios; abertura costal global; tosse); Esclarecer eventuais dúvidas; Avaliar aquisição de conhecimentos da pessoa; Elogiar a pessoa.

Avaliação: A senhora sempre se mostrou recetiva aos ensinos. No decorrer do internamento, ainda que a mesma demonstrasse aquisição completa dos conhecimentos sobre os assuntos abordados, estes foram sempre reforçados. Foi questionada sobre as condições no domicílio, tendo referido que já tinha efetuado as adaptações necessárias. No momento da alta clínica, a senhora encontrava-se apta, demonstrado conhecimento acerca dos cuidados a ter no domicílio, nomeadamente a não realização dos movimentos potencialmente luxantes (flexão acima dos $90^{\circ}$, abdução e rotação externa/interna), a realização dos exercícios isométricos e isotónicos, e a marcha correta e segura com andarilho.

\section{Diagnóstico 2: Dor músculo-esquelética presente à mobilização do membro inferior esquerdo.}

Objetivos: Controlar / diminuir dor; recuperar / manter a funcionalidade; prevenir alterações posturais.

Intervenções: Avaliar e monitorizar a dor usando a escala numérica da dor; Instruir a manter o alinhamento corporal e posições estáveis; Alternar decúbitos; Realizar mobilizações passivas e ativas-assistidas das articulações, dependendo da tolerância da pessoa e segundo os limites articulares; Instruir e treinar a realização de exercícios isométricos e isotónicos do membro operado e segmentos livres, de acordo com a tolerância e limite articular; Gerir 
a atividade e o repouso, de modo a evitar a fadiga e o cansaço muscular; Manter elevação do membro inferior esquerdo; Realizar crioterapia no membro inferior esquerdo; Valorizar a dor da pessoa.

Avaliação: A senhora referia dor intensa no pré-operatório, nível 7, sendo que no pós-operatório, embora referisse dor devido à intervenção cirúrgica, esta já era de nível 5, diminuindo para uma dor 3 nos dias seguintes.

Esta diminuição é justificada pela mobilização precoce do membro intervencionado no pós-operatório. A implementação do programa de reabilitação teve em conta a presença da dor e a sua intensidade, bem como o horário da analgesia prescrita.

\section{Diagnóstico 3: Movimento muscular diminuído no membro inferior esquerdo.}

Objetivos: Promover o fortalecimento muscular e o aumento da amplitude articular; recuperar a funcionalidade; prevenir alterações posturais, atrofias e contraturas musculares.

Intervenções: Executar mobilizações passivas e ativas-assistidas no membro inferior esquerdo e segmentos articulares; Executar técnica de mobilização articular ativa-assistida: flexão e extensão dos dedos dos pés, flexão plantar e dorsiflexão da articulação tibiotársica, flexão e extensão do joelho (flexão até $90^{\circ}$ ), extensão, abdução e adução sem ultrapassar a linha média da articulação coxofemoral; Executar técnica de movimento muscular (exercícios isométricos dos flexores (psoas ilíaco, costureiro, reto anterior e tensor da fáscia lata) e dos extensores (isquiotibiais e grande nadegueiro)) - 3 séries de 10 segundos; Incentivar à realização autónoma dos movimentos musculares e articulares; Supervisionar o movimento muscular; Avaliar conhecimento sobre técnicas de exercício muscular e articular; Realizar levante precoce nas primeiras 24h; Monitorizar amplitude articular e força muscular; Elogiar a pessoa.

Avaliação: Ao aplicar a escala da força de Medical Research Council, a senhora apresentava no pré-operatório (dia 19/11) força $5 / 5$ em todos os segmentos corporais de ambos os membros superiores e membro inferior direito. Apresentava força 5/5 na articulação tibiotársica e joelho esquerdos e força 3/5 na articulação coxofemoral esquerda (realizava movimento ativo, arco de movimento completo contra a gravidade, mas quando era aplicada alguma resistência, havia defesa à dor). No período pós-operatório, nomeadamente no dia 23/11, a força na articulação coxofemoral esquerda situava-se no nível 2/5 (não vencia a gravidade). No entanto, após a aplicação do programa de intervenção de reabilitação, a força aumentou para o nível 4/5, no dia 26/11. Em relação à amplitude articular da articulação coxofemoral esquerda, em extensão manteve-se sempre em $0^{\circ}$. 0 aumento da amplitude articular foi verificado na flexão desta articulação, que aumentou de $30^{\circ}$ no dia $23 / 11$, para $90^{\circ}$ no dia $26 / 11$.

\section{Diagnóstico 4: Equilíbrio corporal comprometido.}

Objetivos: Promover o equilíbrio sentado e ortostático; melhorar postura corporal; recuperar a funcionalidade.

Intervenções: Avaliar equilíbrio corporal recorrendo à escala simples de equilíbrio; Assistir a pessoa para promover equilíbrio através da técnica de posicionamento; Supervisionar o equilíbrio corporal da pessoa: equilíbrio estático e dinâmico sentado e equilíbrio estático e dinâmico ortostático; Estimular a manter o equilíbrio corporal; Executar técnica de posicionamento e de treino de equilíbrio para promover equilíbrio corporal; Instruir e treinar técnica de equilíbrio corporal; Elogiar pessoa; Realizar registos no programa SClínico.

Avaliação: A senhora apresentava equilíbrio sentado (estático e dinâmico) mantido e equilíbrio ortostático (estático e dinâmico) diminuído. Não suportava a posição ortostática por muito tempo, tendo de recorrer ao apoio de mobiliário. No pós-operatório, aplicou-se a escala simples de equilíbrio, tendo-se obtido melhorias relativas ao equilíbrio ortostático e necessidade de correção postural esporádica.

\section{Diagnóstico 5: Capacidade para transferir-se comprometida em grau moderado.}

Objetivos: Promover independência da pessoa; capacitar para o autocuidado.

Intervenções: Avaliar autocuidado transferir-se; Instruir e treinar a técnica de levante do leito (sair pelo lado que costuma sair da cama no domicílio, atendendo a que: o membro operado não pode ultrapassar a linha média do corpo; quando sentada no leito, o tronco não deve fletir, de modo a manter o máximo de flexão da articulação coxofemoral nos $90^{\circ}$ ); Instruir e treinar técnica de transferência para cadeirão / sanitário com o recurso do andarilho (membro inferior esquerdo operado em extensão imediatamente antes de se sentar; uma mão apoiada no andarilho e a outra nas barras de segurança da sanita, baixando-se lentamente e usando a força do membro inferior são e dos membros superiores); Supervisionar no autocuidado transferir-se; Incentivar à realização autónoma dos movimentos musculares e articulares (movimentos isotónicos e isométricos no leito e no cadeirão); Elogiar pessoa; Realizar registos no programa SClínico.

Avaliação: A senhora antes do internamento era previamente independente, embora com alguma limitação e dificuldade na realização das transferências por dor e rigidez articular no membro inferior esquerdo. No período pósoperatório, foi treinada a técnica de transferência e a senhora necessitou apenas de assistência para realizar a 
mesma. Também foi dado ênfase à realização dos exercícios isométricos e isotónicos, de modo a promover a mobilização articular e o fortalecimento muscular. Após o programa, no dia 26/11, a senhora conseguia realizar a técnica de transferência da cama para a posição ortostática, e desta para a cadeira/cadeirão/sanitário, de forma independente.

\section{Diagnóstico 6: Andar com auxiliar de marcha comprometido.}

Objetivos: Promover independência; capacitar para o autocuidado andar com auxiliar de marcha; promover marcha correta e segura.

Intervenções: Avaliar e treinar o equilíbrio: sentado e ortostático (estático e dinâmico); Ensinar e avaliar sobre adaptação do domicílio para andar com auxiliar de marcha; Ensinar sobre os cuidados com o auxiliar de marcha a ser utilizado (ajustar à altura da pessoa; avaliar sinais de desgaste); Instruir e treinar técnica de levante e de sentar com andarilho (realizar extensão do membro inferior esquerdo operado, de modo a realizar carga com os membros superiores no andarilho e na perna direita); Instruir e treinar técnica de marcha a 3 pontos com andarilho, em plano horizontal ( $1^{\circ}$ mobilizar andarilho $15-20 \mathrm{~cm} ; 2^{\circ}$ mobilizar membro inferior esquerdo intervencionado; $3^{\circ}$ mobilizar perna direita para junto da esquerda, sem a ultrapassar ou ao andarilho); Instruir e treinar técnica de inversão do sentido de marcha (de preferência para o lado operado, mas sempre atendendo ao modo como vira o tronco para evitar a rotação interna e externa do membro inferior esquerdo intervencionado); Realizar reeducação ao esforço (realizar consciencialização e dissociação dos tempos respiratórios, bem como pausas durante a marcha); Avaliar o ciclo da marcha da pessoa, tendo em conta a distância alcançada e a duração; Elogiar pessoa; Realizar registos no programa SClínico.

Avaliação: Relativamente a este autocuidado, a senhora rapidamente assimilou o conhecimento sobre a realização de um padrão de marcha correto e seguro. Prévio ao início do treino, avaliou-se a tolerância ao esforço e alertou-se para a possível necessidade de se realizar pausas na marcha. No dia 23/11 necessitou de assistência para a realização do treino de marcha, sob orientação de duas pessoas, com reforço da técnica e correção esporádica da postura, percorrendo cerca de $10 \mathrm{~m}$ e solicitando pausa, retornando ao cadeirão percorrendo mais $10 \mathrm{~m}$. No dia $24 / 11$, a senhora apenas necessitou de supervisão, demonstrando conhecimento adquirido da técnica, bem como das adaptações do domicílio, nomeadamente no que se refere às borrachas do andarilho, à remoção de tapetes e ao alteador de sanita, percorrendo uma distância de cerca de 30m. No dia 26/11, dia da alta, a senhora encontrava-se independente no andar com auxiliar de marcha andarilho, bem como nos cuidados de manutenção do andarilho e calçado apropriado. Manteve o ortostatismo, realizando marcha com recurso a andarilho, sem carga no membro inferior esquerdo (indicação médica), cerca de 50m. Demonstrou assim conhecimento adquirido acerca do padrão de marcha correto e seguro.

\section{Diagnóstico 7: Alto risco de queda presente.}

Objetivos: Prevenir queda; reduzir risco de queda.

Intervenções: Avaliar risco de queda utilizando a escala de quedas de Morse; Otimizar o ambiente físico; Educar sobre manutenção de auxiliares de marcha e apoio na marcha e uso de sapato fechado antiderrapante; Executar medidas de segurança; Avaliar o equilíbrio sentado e ortostático (estático e dinâmico); Realizar treino de equilíbrio ortostático dinâmico; Realizar reforço da técnica e correção postural; Supervisionar técnica de marcha com andarilho, efetuando apoio subaxilar e mantendo uma cadeira de rodas perto da pessoa; Avaliar coordenação motora; Elogiar pessoa; Realizar registos no programa SClínico.

Avaliação: No dia 19/11, o score da escala de Morse foi de 55, indicativo de alto risco de queda. Posteriormente, após a intervenção cirúrgica e levante, o score foi de 60, mantendo-se o risco elevado. Inicialmente a senhora apoiava-se na mobília para caminhar, mas após a cirurgia passou a recorrer ao uso de andarilho e a ter medicação endovenosa. No dia da alta clínica, a senhora mantinha o uso do andarilho para caminhar e já não tinha medicação endovenosa prescrita, mas a sua postura ao andar e na transferência ainda se encontrava debilitada, obtendo-se um score de 40, indicativo de baixo risco de queda. Verificou-se assim, uma diminuição do score nesta escala, do início do internamento até ao momento da alta. A senhora foi instruída a uma técnica de marcha correta e segura, não se verificando nenhum incidente de queda durante o internamento.

\section{RESULTADOS}

A senhora integrou o programa de reeducação funcional motora a partir do dia 19/11/2020, tendo sido os momentos principais de aplicação dos instrumentos de avaliação nos dias 23 e 26/11/2020. No decorrer do programa, a senhora manteve-se sempre hemodinamicamente estável, tendo solicitado medicação analgésica complementar para colmatar, tanto as dores da coxartrose esquerda, como posteriormente para reduzir a dor resultante do pósoperatório. Relativamente à avaliação do padrão respiratório, verificou-se um padrão rítmico com amplitudes regulares, sendo que à auscultação pulmonar o murmúrio vesicular encontrava-se mantido bilateralmente e sem ruídos adventícios, apesar do antecedente pessoal de asma. 
De modo a verificar-se que efetivamente a senhora se encontrou consciente e orientada, recorreu-se à escala de coma de Glasgow, obtendo-se sempre a pontuação máxima de 15 durante o internamento. Desta forma, os ensinos pré-operatórios tiveram um efeito positivo e benéfico, ao permitirem diminuir a ansiedade sentida. Na literatura, verifica-se que as maiores preocupações relatadas consistem no medo pelo desconhecido e na dor que irá ser sentida $^{(3)}$. Neste sentido, a importância da aplicação desta escala é justificada no momento dos ensinos, que são necessários realizar à senhora e para os quais há a necessidade de que esta esteja disposta e desperta para tal, de modo a permitir que compreenda os cuidados pós-operatórios e possa dar-lhes continuidade no domicílio.

Tendo em conta a aplicação da escala de força de Medical Research Council, não houve alterações em termos de força dos segmentos corporais dos membros superiores e membro inferior direito. Apenas se verificou alterações na articulação coxofemoral do membro inferior esquerdo, que foi submetido a intervenção cirúrgica. Inicialmente, no que se refere à articulação coxofemoral do membro inferior esquerdo, este apresentava força $3 / 5$, pois a senhora conseguia vencer a gravidade, algo que já não ocorria no pós-operatório, apresentando uma força $2 / 5$ no dia $23 / 11 / 2020$. No dia 26 , dia da alta, foi alcançada força $4 / 5$, definida pela capacidade de vencer a gravidade e na aplicação de uma moderada resistência.

Em relação à avaliação da amplitude articular com goniómetro, registou-se apenas as amplitudes da articulação coxofemoral do membro inferior esquerdo, uma vez que as demais articulações se encontravam dentro dos valores de amplitude articular fisiológica. Não foram avaliados a rotação interna ou externa, nem a adução para além da linha média, uma vez que representam movimentos luxantes a evitar. No dia 19/11/2020 a senhora recusou a mobilização do membro inferior esquerdo para avaliação da amplitude articular da articulação coxofemoral devido a dor intensa. Ainda assim, após a cirurgia pode-se verificar melhorias do dia 23 para o dia 26 , uma vez que houve um aumento das amplitudes articulares deste membro. A flexão passou de $30^{\circ}$ para $90^{\circ}$ e a abdução de $25^{\circ}$ para $29^{\circ}$. 0 goniómetro constitui assim um instrumento que possibilita uma avaliação com boa confiabilidade das amplitudes de movimento, tanto das articulações dos membros inferiores, como dos superiores ${ }^{(20)}$.

Relativamente à escala simples de equilíbrio, a senhora manteve sempre o equilíbrio sentado estático e dinâmico. Antes de ser intervencionada cirurgicamente, apresentava equilíbrio ortostático estático e dinâmico diminuído, uma vez que se apoiava no mobiliário para conseguir manter o ortostatismo e deambular. Após a cirurgia, verificou-se uma melhoria a nível do equilíbrio ortostático estático e dinâmico.

De acordo com a Escala de Barthel, pôde-se verificar que, tanto no pré-operatório, como no pós-operatório, a senhora apresentou sempre uma moderada dependência, que se manteve no dia da alta. Ainda assim, no momento da alta, a pontuação foi de 90, limiar da dependência muito leve, e superior tanta à avaliação do dia 19/11, como da de dia 23/11 (pontuação de 75 e 65 respetivamente). No dia da alta, a senhora já percorria mais de 50 metros com o auxiliar de marcha andarilho, sem pausas e de forma independente.

Em relação à escala de quedas de Morse, a senhora deu entrada no serviço com um alto risco de queda. No momento da alta clínica foi obtido ume score indicativo de baixo risco de queda, podendo ser justificado, não só pela intervenção cirúrgica em si, como pelo acompanhamento do EEER.

Relativamente à dor e recorrendo à escala numérica ${ }^{(19)}$, verificou-se que a dor era muito superior antes da cirurgia. Após a intervenção cirúrgica, a senhora também referiu dor, mas em menor intensidade.

Quadro 1 - Síntese dos resultados obtidos

\begin{tabular}{|l|c|c|c|}
\hline Resultados obtidos & Dia 19 & Dia 23 & Dia 26 \\
\hline Escalas & 15 & 15 & 15 \\
\hline Escala de Coma de Glasgow & $3 / 5$ & $2 / 5$ & $4 / 5$ \\
\hline $\begin{array}{l}\text { Escala de Força de Medical Research Council } \\
\text { (coxa esquerda) }\end{array}$ & - & $\begin{array}{l}\text { F: } 30^{\circ} \\
\text { A: } 25^{\circ}\end{array}$ & $\begin{array}{c}\text { F: } 90^{\circ} \\
\text { A: } 29^{\circ}\end{array}$ \\
\hline Amplitude Articular - Goniómetro (coxofemoral esquerda) & E e D: Dim & $\begin{array}{c}\text { E: Man } \\
\text { D: Dim }\end{array}$ & E e D: Man \\
\hline Escala Simples de Equilíbrio (ortostático) & 75 & 65 & 90 \\
\hline Escala de Barthel & 55 & 60 & 40 \\
\hline Escala de Quedas de Morse & 7 & 3 & 2 \\
\hline Escala Numérica da Dor & & & \\
\hline
\end{tabular}

Legenda: F-Flexão; A - Abdução; E-Estático; D - Dinâmico; Dim - Diminuído; Man - Mantido.

\section{DISCUSSÃO}

Todo e qualquer programa de reabilitação deve abordar a pessoa desde o pré-operatório, esclarecendo-a sobre a intervenção cirúrgica e os seus objetivos, sinais e sintomas de possíveis complicações e demonstrar os ganhos de uma 


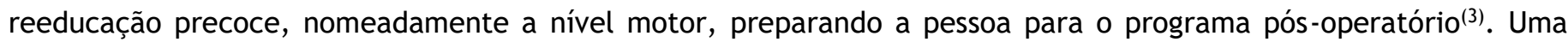
capacitação eficaz deve assegurar a satisfação das necessidades da pessoa na transição de cuidados, permitindo melhorar a sua funcionalidade ${ }^{(21)}$. A senhora de que trata o presente estudo de caso demonstrou conhecimento adquirido no pós-operatório, o que ressalva a intervenção do EEER e a importância da sua atuação precoce no período pré-operatório. Embora não tenha sido possível capacitar o cuidador informal devido à situação de pandemia mundial, no seu estudo, Huang et al. verificam que a implementação de um programa de empoderamento em pessoas submetidas a artroplastia total da anca foi eficaz em termos de melhoria dos resultados e ganhos obtidos, os quais teriam tido um maior impacto se os cuidadores informais participassem no programa. Deste modo, as intervenções direcionadas para a educação para a saúde têm demonstrado ganhos no desempenho das atividades de vida diária, mobilidade e qualidade de vida(22).

Os principais objetivos do tratamento cirúrgico e da intervenção do EEER prendem-se ao alívio da dor e à melhoria da capacidade funcional e qualidade de vida, o que promove consequentemente a mobilidade articular e evita a redução da massa muscular ${ }^{(4)}$.

O procedimento cirúrgico da artroplastia da anca relaciona-se com uma melhoria da funcionalidade da pessoa, nomeadamente no que concerne à diminuição da dor ${ }^{(23)}$. Esta afirmação pode ser verificada no presente estudo de caso, pois houve uma diminuição gradual da dor, no decorrer do internamento. A senhora transitou de uma dor de nível 7 incapacitante no pré-operatório, para uma dor de nível 2 no dia da alta, ou seja, verificou-se uma melhoria na sua qualidade de vida. A realização de um programa de reabilitação no pós-operatório também possibilita melhorar, a curto prazo, a qualidade de vida das pessoas ${ }^{(20)}$. Ainda assim, esta diminuição de dor pode estar associada a diversos aspetos, tais como a intervenção cirúrgica em si, o uso de auxiliares de marcha, o repouso ou a administração de analgesia, pelo que se torna um desafio atribuir a principal causa para esta melhoria sentida no período pós-operatório ${ }^{(23)}$.

Existem estudos feitos em Portugal que constatam melhorias significativas, não só em termos de qualidade de vida, como a nível de independência funcional, mobilidade e equilíbrio, como resultado da intervenção do EEER ${ }^{(20)}$.

Um programa de treino adicional e específico de mobilização articular e de força dos músculos da anca, desenvolvido por Matheis e Stöggl e implementado no $3^{\circ}$ dia de pós-operatório em pessoas submetidas a artroplastia total da anca, concluiu que, além de ser bem tolerado pelos doentes, numa semana há melhorias relativas à amplitude articular e marcha, quando comparados com a fisioterapia convencional ${ }^{(6)}$.

Esta afirmação vai de encontro aos resultados obtidos no presente estudo de caso, pois a partir da avaliação do goniómetro verificaram-se diferenças de $60^{\circ}$ na flexão e de $4^{\circ}$ na abdução da articulação coxofemoral; e melhorias no equilíbrio ortostático estático e dinâmico, ambos imprescindíveis no desempenho da marcha. Deste modo, numa cirurgia eletiva de artroplastia total da anca, a reabilitação precoce, iniciada no pré-operatório, contribui de forma significativa para a mobilidade, força muscular e dor, existindo ainda ganhos pontuais nos valores goniométricos de determinados movimentos ${ }^{(23)}$.

A pessoa pode recuperar mais rapidamente a sua independência para realizar as atividades de vida diária, assim que superar o défice na marcha, ou seja, as intervenções direcionadas para reabilitar a pessoa com andar comprometido, promovem a sua independência ${ }^{(24)}$. No presente trabalho, ainda que a senhora tenha mantido o grau de dependência moderada na Escala de Barthel, houve um aumento na pontuação no dia da alta, o que remete para a total independência em alguns itens da escala, nomeadamente no da transferência e da mobilidade. Assim, conclui-se que a escala de Barthel é determinante na avaliação da capacidade funcional da pessoa submetida a artroplastia total da anca por coxartrose, uma vez que pessoas independentes possuem uma maior capacidade funcional(23).

A partir da mobilização da pessoa idosa submetida a artroplastia da anca obtém-se uma melhoria da sua capacidade funcional. Além deste ganho em saúde resultante da intervenção do enfermeiro, a mobilização da pessoa submetida a artroplastia da anca também permite uma maior independência na sua mobilidade, nomeadamente nas mobilizações no leito, nas transferências e na deambulação com ou sem auxiliar de marcha ${ }^{(25)}$.

Inteiramente relacionado com o treino de marcha destaca-se a segurança da pessoa e a prevenção de quedas, onde se aplica a escala de quedas de Morse. Importa assim realçar a utilização de calçado apropriado, a diminuição das barreiras arquitetónicas e de tapetes, avaliando deste modo todo o ambiente circundante ${ }^{(24)}$. A senhora teve alta com um baixo risco de queda, tendo contribuído para este valor o facto de já não ter medicação endovenosa, pois embora estivesse consciente das suas capacidades, ainda necessitava do andarilho para caminhar, apresentava uma postura debilitada no andar e transferência, bem como o diagnostico secundário de asma e coxartrose.

No que se refere à mobilidade articular, tanto os valores obtidos pelo goniómetro, como os valores de força obtidos pela escala de força da Medical Research Council são importantes e determinantes para avaliar a capacidade funcional da pessoa, caracterizados por maiores amplitudes de movimento articular e elevados valores de força muscular(23). Os dados obtidos vão de encontro ao estudo de Winther et al., que objetivou a maximização do treino de força em doentes submetidos a artroplastia total da anca, concluindo ser um programa bem tolerado pelos doentes e de fácil integração e aplicação no contexto da prática clínica, com a obtenção de ganhos favoráveis no desenvolvimento da força muscular até 6 meses de pós-operatório, após 3 meses de reabilitação comparativamente à fisioterapia convencional ${ }^{(26)}$. 
Outro estudo elaborado por Mitrovic et al., demonstrou que exercícios suplementares dos membros superiores e parte superior do tronco contribuíram para ganhos na recuperação da força muscular, em doentes idosos, 12 semanas após a intervenção cirúrgica, o que contribuiu igualmente para a melhoria da qualidade de vida relacionada com a saúde ${ }^{(27)}$.

Uma análise de estudos de 2009 a 2018 demonstrou que as pessoas submetidas a artroplastia total da anca e intervenção do EEER evidenciam uma maior independência funcional, equilíbrio, mobilidade, e por isso, mais qualidade de vida. De um modo mais específico, pode-se particularizar no caso das pessoas com prótese total da anca, que a intervenção do EEER na realização de sessões de educação para a saúde no momento pré e pós-operatório produz melhorias significativas relativamente à dor, independência no desempenho das atividades de vida diária, amplitude de movimento e marcha. Estas melhorias correspondem por sua vez aos resultados obtidos no presente estudo de caso $^{(28)}$.

Desta forma, verifica-se que os EEER assumem um papel preponderante na implementação de programas de reabilitação de treino motor e de atividades de vida diária, que devem ir de encontro aos objetivos, diagnósticos e necessidade da pessoa, com vista à sua máxima recuperação funcional(20).

\section{Perspetiva da pessoa}

A senhora sempre se mostrou muito recetiva aos cuidados prestados, agradecendo pelo tempo despendido e pela atenção, preocupação e calma com que foram explicadas as técnicas e exercícios musculo-articulares. Referiu ter esclarecido as suas dúvidas sempre que estas surgiam, e que se encontrava menos preocupada com o regresso a casa, comparativamente com o início do internamento.

\section{CONCLUSÃO}

O presente estudo de caso permite confirmar a importância do papel do EEER na implementação de um programa de reabilitação específico para a promoção da independência funcional da pessoa submetida a artroplastia total da anca, destacando-se a reeducação funcional motora e treino de atividades de vida diária.

A senhora em estudo apresentou uma evolução favorável ao longo do internamento, tendo contribuído a intervenção do EEER. Houve necessidade de recorrer a instrumentos de avaliação que permitissem avaliar de uma forma holística determinadas funções da pessoa, tais como: estado de consciência, força muscular, amplitude articular, equilíbrio, nível de dependência, risco de queda e intensidade da dor. Os dados resultantes destes instrumentos de avaliação auxiliaram na elaboração dos diagnósticos de enfermagem, assim como na monitorização das intervenções implementadas e avaliação dos resultados obtidos. Ao longo da implementação deste programa de reabilitação, verificou-se uma melhoria gradual na independência funcional da senhora na realização das atividades de vida diária, comparativamente à data de admissão. Deste modo, os dados obtidos e sintetizados no quadro 1 possibilitam destacar a relevância do EEER e a sua intervenção com a pessoa com alterações e necessidades especiais do foro músculoesquelético, tendo-se verificado melhorias a nível da força muscular da coxa esquerda, da amplitude articular da articulação coxofemoral esquerda, no equilíbrio corporal, na independência para a realização das atividades de vida diária e no risco de queda.

Como limitações ao estudo identifica-se: a dificuldade da senhora em cumprir os treinos na ausência do EEER e o facto da dor referida durante a implementação do programa constituir um fator limitador na aplicação de certas intervenções. Como se trata de um relato de caso em concreto, e deste modo de uma pessoa com determinado diagnóstico específico, os resultados obtidos não podem ser generalizados, mas sim servir para o desenvolvimento do conhecimento neste contexto, e permitir melhorar o planeamento e a implementação das intervenções do EEER nestas situações específicas da prática clínica. Desta forma, o presente trabalho consiste numa estratégia de pesquisa científica, que permite investigar certos fenómenos no contexto real da prática de enfermagem, sua reflexão e procura por novas soluções aos problemas evidenciados, o que contribui inevitavelmente para o desenvolvimento do conhecimento $^{(29)}$.

Devido à pandemia mundial por SARS-CoV-2 verificou-se outra limitação neste estudo de caso, que foi a capacitação do cuidador informal, algo que não foi possível realizar devido à restrição de visitas imposta no internamento.

Por outro lado, também constituiu uma limitação ao estudo o facto de parte da literatura e estudos internacionais encontrados não definirem claramente as intervenções específicas de enfermagem de reabilitação, pelo que houve a necessidade de utilizar estudos que recorressem a intervenções ou programas de reabilitação adotados por outros profissionais, cuja intervenção remetesse para competências do EEER.

Como sugestões de práticas futuras destaca-se a importância de se continuar a realizar planos de cuidados integrados em estudos de caso, baseados na e para produzir evidência científica, de modo a permitir a melhoria contínua da qualidade dos cuidados prestados e o aperfeiçoamento profissional.

\section{REFERÊNCIAS BILIOGRÁFICAS}

1. Fernandes MJ. Envelhecimento bem-sucedido: modelo de intervenção da enfermagem. Em: Lopes MA, organizador. O cuidado de enfermagem 
à pessoa idosa: da investigação à prática. Loures: Lusociência; 2013. p. 3-37.

2. Instituto Nacional de Estatística. Estatísticas da Saúde - 2019. [Site web] Lisboa: Instituto Nacional de Estatística; 2021 [citado em 27 de maio de 2021].

Disponível

https: / / www.ine.pt/xportal/xmain?xpid=INE\&xpgid=ine_publicacoes\&PUBLICACOESpub_boui=257483090\&PUBLICACOESmodo=2

3. Parente C, Ângelo M, Tinoco A, Rocha E, Brandão D, Cristina M, Céu M. Artroplastia da anca. Cruz AG, Conceição VC, Oliveira LM, coordenadores. Enfermagem em ortotraumatologia. Coimbra: Formasau; 2009. p. 129-163.

4. Colibazzi V, Coladonato A, Zanazzo M, Romanini E. Evidence based rehabilitation after hip arthroplasty. HIP International. 2020; 30(2S): 2029. Diponível em: https://doi.org/10.1177/1120700020971314

5. Winther SB, Foss OA, Klaksvik J, Husby VS. Increased muscle strength limits postural sway during daily living activities in total hip arthroplasty patients. American Journal of Physical Medicine \& Rehabilitation. 2020; 99(7): 608-612. Disponível em: https://doi.org/10.1097/PHM.0000000000001382

6. Matheis C, Stöggl T. Strength and mobilization training within the first week following total hip arthroplasty. Journal of Bodywork \& Movement Therapies. 2017. Disponível em: https://doi.org/10.1016/j.jbmt.2017.06.012

7. Ordem dos Enfermeiros. Regulamento das competências específicas do enfermeiro especialista em enfermagem de reabilitação. [Site web] Ordem dos Enfermeiros; 2010 [atualizado em 2010; citado em 20 de novembro de 2020]. Disponível em: https://www.ordemenfermeiros.pt/arquivo/legislacao/Documents/LegislacaoOE/RegulamentoCompetenciasReabilitacao_aprovadoAG20Nov 2010.pdf

8. Andrade SR, Ruoff AB, Piccoli T, Schmitt MD, Ferreira A, Xavier AC. O estudo de caso como método de pesquisa em enfermagem: uma revisão integrativa. Texto \& Contexto Enfermagem. 2017; 26(4): 1-12. Disponível em: https://doi.org/10.1590/0104-07072017005360016

9. Riley DS, Barber MS, Kienle GS, Aronson JK, Schoen-Angerer T, Tugwell P ... Gagnier JJ. CARE guidelines for case reports: explanation and elaboration document. J Clin Epidemiol. 2017; 89: 218-35. Disponível em: https://doi.org/10.1016/j.jclinepi.2017.04.026

10. Conselho Internacional de Enfermeiros. CIPE $®$ Versão 2015 - classificação internacional para a prática de enfermagem. Lusodidacta; 2016.

11. Ordem dos Enfermeiros. Padrão documental dos cuidados de enfermagem da especialidade de enfermagem de reabilitação. [Site web] Ordem dos Enfermeiros; 2014 [atualizado em 2014; citado em 19 de novembro de 2020]. Disponível em: https://www.ordemenfermeiros.pt/arquivo/colegios/Documents/2015/MCEER_Assembleia/PadraoDocumental_EER.pdf

12. Santos LL. 0 processo de reabilitação. Em: Marques-Vieira C, Sousa L, coordenadores. Cuidados de enfermagem de reabilitação à pessoa ao longo da vida. Loures: Lusodidacta; 2016. p. 15-23.

13. Sousa L, Marques-Vieira C, Severino S, Caldeira S. Propriedades psicométricas de instrumentos de avaliação para a investigação e prática dos enfermeiros de reabilitação. Em: Marques-Vieira C, Sousa L, coordenadores. Cuidados de enfermagem de reabilitação à pessoa ao longo da vida. Loures: Lusodidacta; 2016. p. 113-121.

14. Jennett B, Teasdale G. Glasgow coma scale/score (GCS). [Site web] MDCalc; 2020 [atualizado em 2020; citado em 19 de novembro de 2020]. Disponível em: https://www.mdcalc.com/glasgow-coma-scale-score-gcs\#use-cases

15. Medical Research Council. Aids to the examination of the peripheral nervous system. [Site web] Medical Research Council; 1976 [citado em 19 de novembro de 2020]. Disponível em: https://mrc.ukri.org/documents/pdf/aids-to-the-examination-of-the-peripheral-nervous-system-mrcmemorandum-no-45-superseding-war-memorandum-no-7

16. Shah S, Vanclay F, Cooper B. Improving the sensitivity of the Barthel Index for stroke rehabilitation. Journal of Clinical Epidemiology. 1989; 42(8): 703-708. Disponível em: https://doi.org/10.1016/0895-4356(89)90065-6

17. Direção-Geral da Saúde. Norma 008/2019 - Prevenção e Intervenção na Queda do Adulto em Cuidados Hospitalares. [Site web] Lisboa: Direção Geral da Saúde; 2019 [atualizado em 2020; citado em 19 de novembro de 2020]. Disponível em: https://normas.dgs.min-saude.pt/wpcontent/uploads/2019/12/prevencao-e-intervencao-na-queda-do-adulto-em-cuidados-hospitalares.pdf

18. Dias MJMC, Ferreira PL. Escalas de avaliação de risco de quedas. Revista de Enfermagem Referência. 2014; IV(2): 153-161. Disponível em: http: //dx.doi.org/10.12707/RIII12145

19. Direção-Geral da Saúde. A Dor como $5^{\circ}$ sinal vital - Registo sistemático da intensidade da dor. [Site web] Lisboa: Direção Geral da Saúde; 2003 [atualizado em 2020; citado em 19 de novembro de 2020]. Disponível em: https://www.dgs.pt/directrizes-da-dgs/normas-e-circularesnormativas/circular-normativa-n-9dgcg-de-14062003-pdf.aspx

20. Sousa L, Carvalho ML. Pessoa com osteoartrose na anca e joelho em contexto de internamento e ortopedia. Em: Marques-Vieira C, Sousa L, coordenadores. Cuidados de enfermagem de reabilitação à pessoa ao longo da vida. Loures: Lusodidacta; 2016. p. 405-420.

21. Sousa L, Carvalho ML. Pessoa com fratura da extremidade superior do fémur. Em: Marques-Vieira C, Sousa L, coordenadores. Cuidados de enfermagem de reabilitação à pessoa ao longo da vida. Loures: Lusodidacta; 2016. p. 421-431.

22. Huang $T$, Sung CC, Wang WS, Wang BH. The effects of the empowerment education program in older adults with total hip replacement surgery. Journal of Advanced Nursing. 2017; 73(8): 1848-1861. Disponível em: https://doi.org/10.1111/jan.13267

23. Gonçalves LM. Determinantes da capacidade funcional em indivíduos com coxartrose submetidos a ATA. Viseu: Escola Superior de Saúde do Instituto Politécnico de Viseu; 2014 [citado em 22 de novembro de 2020]. Disponível em: https://repositorio.ipv.pt/handle/10400.19/2560

24. Marques-Vieira C, Caldas AC. A relevância do andar: reabilitar a pessoa com andar comprometido. Em: Marques-Vieira C, Sousa L, coordenadores. Cuidados de enfermagem de reabilitação à pessoa ao longo da vida. Loures: Lusodidacta; 2016. p. 547-557.

25. Pereia I, Ferreira RF, Vieira JV, Goes MM, Mestre T, Oliveira H. Gains from nursing care in mobilizing the elderly person after hip arthroplasty. In: Garcia-Alonso J, Fonseca C, editores. Gerontechnology III. Contributions to the Third International Workshop on Gerontechnology, IWoG 2020; 2020 Out 5-6; Évora, Portugal. Suiça: Springer Nature; 2021: 262-276. Disponível em: https://doi.org/10.1007/978-3-030-72567-9_25

26. Winther SB, Foss OA, Husby OS, Wik TS, Klaksvik J, Husby VS. A randomized controlled trial on maximal strength training in 60 patients undergoing total hip arthroplasty. Acta Orthopaedica. 2018; 89. Disponível em https://doi.org/10.1080/17453674.2018.1441362

27. Mitrovic D, Davidovic M, Erceg P, Marinkovic J. The effectiveness of supplementary arm and upper body exercises following total hip arthroplasty for osteoarthritis in the elderly: a randomized controlled trial. Clinical Rehabilitation. 2016; 1-10. Disponível em: https: //doi.org/10.1177/0269215516655591

28. Santos MFP. Prótese total da anca: efetividade e um programa de reabilitação. Viseu: Escola Superior de Saúde do Instituto Politécnico de Viseu; 2019 [citado em 30 de maio de 2021]. Disponível em: https://repositorio.ipv.pt/handle/10400.19/5455

29. Silva LAGP, Mercês NNA. Estudo de casos múltiplos aplicado na pesquisa de enfermagem: relato de experiência. Revista Brasileira de Enfermagem. 2018; 71(3): 1194-7. Disponível em: http://dx.doi.org/10.1590/0034-7167-2017-0066 


\section{DIVULGAÇÕES ÉTICAS}

Contribuição do(s) autor(es):

Conceptualização: PD, PM, RFF;

Metodologia: PD, RFF;

Validação: PD, PM, RFF;

Análise formal: $P D, R F F$;

Investigação: PD, PM, RFF;

Tratamento de dados: PD, PM;

Preparação do rascunho original: PD, RFF;

Redação e edição: PD, PM, RFF;

Revisão: PD, PM, RFF;

Todos os autores leram e concordaram com a versão publicada do manuscrito.

Financiamento:

Este trabalho não recebeu nenhuma contribuição financeira ou bolsa.

Declaração de consentimento informado:

0 consentimento informado, livre e esclarecido por escrito foi obtido do participante para publicar este trabalho.

Conflitos de interesse:

Os autores não declaram nenhum conflito de interesses.

\section{Proveniência e revisão por pares:}

Não comissionado; revisto externamente por pares.

(c) Autor (es) (ou seu (s) empregador (es)) e APER/RPER 2021. Reutilização permitida de

(c) acordo com CC BY-NC-ND. Nenhuma reutilização comercial. 